\title{
Implementation of Bethesda system in thyroid aspirate: A cyto-histopathological correlation study
}

\author{
Choudhary PK ${ }^{1}$, Nepal N ${ }^{1}$, Mainali N ${ }^{1}$, Meenakshi B ${ }^{2}$ \\ ${ }^{I}$ Department of Pathology, Nobel Medical College Teaching Hospital and Research Centre, Biratnagar, Nepal. \\ ${ }^{2}$ Department of ENT, Nobel Medical college Teaching Hospital and Research Centre, Biratnagar, Nepal.
}

\section{Keywords:}

Bethesda system;

FNAC;

Thyroid

\begin{abstract}
Background: Thyroid lump is a common clinical problem in Nepal. The risk of malignancy has to be judged prior to surgery for which fine needle aspiration cytology is widely used. To bring the uniform reporting of thyroid aspirates, the Bethesda system for reporting thyroid cytopathology (TBSRTC) was introduced in 2007. This study was done to evaluate the various cytomorphological patterns of thyroid aspirate and to correlate the Bethesda system with final histopathology report.
\end{abstract}

Materials and Methods: Fine needle aspiration were performed and were evaluated as per TBSRTC criteria. Fine needle aspiration cytology report were compared with biopsy specimen whenever possible.

Results: A total of 386 cases were studied. The sensitivity, specificity, positive predictive value, negative predictive value and overall accuracy of TBSRTC were $75.2 \%, 98.2 \%, 90.0 \%, 94.7 \%$ and $94.03 \%$ respectively.

Conclusion: Use of TBSRTC in reporting thyroid cytopathology has a greater accuracy; however, benign cases need regular follow up as there are false negative cases.

\section{INTRODUCTION}

Thyroid lump is a common clinical presentation with a reported prevalence rate of 4 to $7 \% .{ }^{1}$ Benign lesion is more common than malignant ones. ${ }^{1}$ It is very important to establish the nature of lesion to prevent unnecessary surgery which can cause hypothyroidism, hypoparathyroidism and laryngeal nerve palsy. ${ }^{2}$

Several imaging techniques are available for the evaluation

\section{Correspondence:}

Dr. Prabesh Kumar Choudhary, MBBS, MD

Department of Pathology

Nobel Medical College Teaching Hospital and Research Centre

Email:khushbudev.dev@gmail.com of thyroid lump. Fine needle aspiration cytology is still the first diagnostic tool to establish the nature of thyroid lump as it is very rapid, easy, cost effective, minimally invasive out-patient based diagnostic tool which is well tolerated among people and moreover it has a greater accuracy. ${ }^{3}$

In past, terminology regarding thyroid fine needle aspiration cytology (FNAC) has varied significantly from one laboratory to another creating confusion among clinicians working at multiple institutes. The Bethesda System for Reporting Thyroid Cytopathology (TBSRTC) was introduced in 2007 to bring the uniformity among the pathologists in the reporting of thyroid FNAC., ${ }^{45}$ 
This study was done to find out the diagnostic role of TBSRTC in the evaluation of thyroid lump. Cytomorphological patterns and epidemiological profiles of individuals presenting with thyroid lump were also observed.

\section{MATERIALS AND METHODS}

This was a cross-sectional observational study conducted from January 2013 to September 2015. All patients presenting with thyroid lump and who were advised for FNAC were included in this study. Permission from ethical committee was obtained. A written consent was taken in every case. FNAC was performed with 21 gauge needle and smears were stained with May-Grünwald-Giemsa (MGG) and Papanicolaou (Pap) stains as per the standard guidelines.6 Smears were examined by three pathologists and were categorized according to the TBSRTC which categorize the thyroid aspirate into six groups; Non diagnostic (1), benign (2), atypical follicular lesion of undetermined significance (3), follicular neoplasm (4), suspicious for malignancy (5), and malignant (6). ${ }^{4}$ Nondiagnostic category included sample with inadequate material, poor quality smear and that containing cyst fluid only $^{5}$. Categories 2, 3 and 4 were considered cytologically benign and categories 5 and 6 were considered malignant. Histopathological report was considered as the gold standard. All datas were inserted in SPSS 17 software. All cases were followed up and histopathological report was obtained wherever possible. Cytological report was correlated with final histopathological opinion. All cases of malignant histopathological diagnosis in cases of Bethesda 2, $3 \& 4$ categories were considered false negative whereas benign histopathological diagnosis in Bethesda category 5 \& 6 were considered false positive. Diagnostic accuracy of TBSRTC was evaluated by following formulas:

\section{RESULTS}

This was a hospital based prospective cross sectional study. A total of 386 cases were included, out of which 367 were female and 19 were male with age ranging from 10 to 89 years. Thyroid lumps were found most common in 21 to 40 years of age group.

In two of the cases, aspiration material was non-diagnostic and was excluded from the statistical analysis. Benign category was the most common $(90.0 \%)$ followed up by malignant (3.6\%) and follicular neoplasm (3.1\%) categories (Table 1). Non-neoplastic lesions accounted for 347 cases of which colloid goiter was the most common lesion (59.4\%) followed by nodular goiter (12.4\%) and lymphocytic thyroiditis (12.1\%) (Table 2).

Histopathological diagnosis was available in 67 cases who had undergone FNAC. Table 3 shows cyto-histopathological correlation of thyroid lump and the incidence of malignancy in various TBSRTC categories. Incidence of malignancy
Table 1: Distribution of FNAC finding according to TBSRTC categories

\begin{tabular}{lcc}
\hline \multicolumn{1}{c}{ Diagnostic category } & Number & $\begin{array}{c}\text { Percentage } \\
(\mathbf{\%})\end{array}$ \\
\hline Benign & 347 & 90.0 \\
$\begin{array}{l}\text { Atypical follicular lesion of } \\
\text { undetermined significance }\end{array}$ & 09 & 2.3 \\
Follicular neoplasm & 12 & 3.1 \\
Suspicious for malignancy & 04 & 1.0 \\
Malignant & 14 & 3.6 \\
Total & $\mathbf{3 8 6}$ & $\mathbf{1 0 0}$
\end{tabular}

Table 2: Distribution of non-neoplastic thyroid lesion according to cytological diagnosis

\begin{tabular}{lcc}
\hline \multicolumn{1}{c}{ Diagnosis } & Number & $\begin{array}{c}\text { Percentage } \\
(\%)\end{array}$ \\
\hline Colloid goiter & 206 & 59.4 \\
\hline Nodular goiter & 43 & 12.4 \\
\hline Hyperplastic goiter & 36 & 10.4 \\
\hline Lymphocytic thyroiditis & 42 & 12.1 \\
\hline Dequervain's thyroiditis & 10 & 2.9 \\
\hline Thyroglossal cyst & 09 & 2.6 \\
Extramedullary hematopoiesis & 01 & 0.2 \\
Total & $\mathbf{3 4 7}$ & $\mathbf{1 0 0}$
\end{tabular}

was highest in category 5 and $6(90 \%)$ followed by category $4(11.1 \%)$ and $3(10 \%)$. All malignant cases detected on cytological study were that of papillary thyroid carcinoma. The sensitivity, specificity, positive predictive value, negative predictive value and overall accuracy were $75.2 \%$, $98.2 \%, 90.0 \%, 94.7 \%$ and $94.03 \%$ respectively. The risk of malignancy in categories 4,3 and 2 were $11.1 \%, 10 \%$ and $2.6 \%$ respectively.

\section{DISCUSSION}

Enlargement of thyroid is a common clinical presentation which needs diagnostic evaluation prior to surgical intervention. Several diagnostic methods including ultrasonography, radioisotope scan and others are available. Various studies show that FNAC is the most accurate test for the rapid diagnosis of the thyroid lump..$^{3,7}$ Moreover; FNAC is cost effective, easy, and well tolerated among individuals. However, FNAC of thyroid lump has several limitations including inadequate sampling and most important being in its inability to differentiate between benign and malignant follicular lesions in the absence of nuclear features of PTC. Most of these indeterminate lesions comprised of adenomatoid nodule, follicular adenoma, follicular carcinoma and follicular variant of Papillary thyroid carcinoma.

In this study there was predominance of thyroid lump in 
Table 3: Comparison of Cytological diagnosis with final histopathology with remarks and incidence of malignancy in each Bethesda Category $(n=67)$

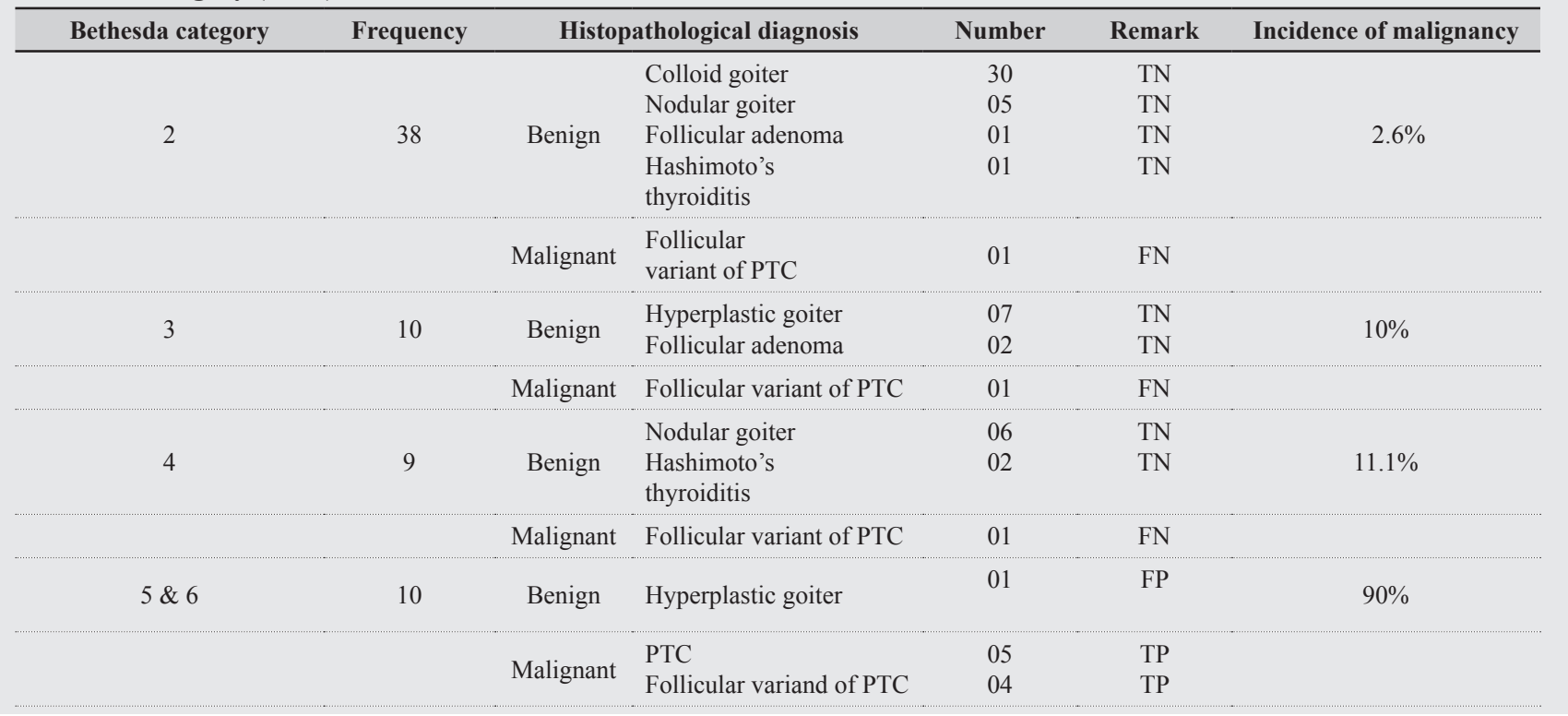

PTC=Papillary thyroid carcinoma; TN: True negative; FN: False negative, TP: True positive

females giving a male to female ratio of $1: 19.3$. Benign lesions were more common than malignant ones. Similar findings were also observed in other studies. ${ }^{8}$

As in other studies, the commonest benign categories were colloid goiter followed up by nodular goiter and lymphocytic thyroiditis. ${ }^{1,8}$ Most common malignant diagnosis was that of papillary thyroid carcinoma which is congruent to other studies. ${ }^{1,9}$

Statistical analysis in our study revealed a sensitivity, specificity, positive predictive value, negative predictive value and accuracy of $75.2 \%, 98.2 \%, 90.0 \%, 94.7 \%$ and $94.03 \%$ respectively. These results were comparable to the others where the FNAC of thyroid has reported sensitivity ranging from $60 \%$ to $99 \%$, specificity $80 \%$ to $100 \%$, positive predictive value $85 \%$ to $97 \%$, negative predictive value $88 \%$ to $99 \%$ and accuracy $89 \%$ to $99 \% .^{1,3,4,8,10}$ Reasons for these varied results may be due to number of cases, subjective errors and sampling errors.

Risk of malignancy among the various non-malignant categories was highest in category 4 followed by categories 3 and 2. These cases should therefore be followed up to detect any progression to malignancy. Other study show the risk of malignancy to be highest in category ${ }^{3,11}$ This variation can be due to subjective error and number of cases included in the study. The overall accuracy of $94 \%$ in our study is comparable to others and certainly confirms the diagnostic role of TBSRTC in the reporting of thyroid lump.

\section{CONCLUSION}

The Bethesda system for reporting thyroid cytopathology has a greater accuracy and therefore we recommend routine use of TBSRTC for reporting thyroid aspirate. However, non-malignant cytological interpretation should be viewed with caution as there are false negative cases and thus these cases should undergo regular clinico-radiological follow up to look for any progression that will require repeat FNAC and/or surgical intervention.

\section{REFERENCES}

1. Sinna EA, Ezzat N. Diagnostic accuracy of fine needle aspiration cytology in thyroid lesions. Journal of the Egyptian National Cancer Institute. 2012;24:63-70. Crossref

2. De Palma M, Rosato L, Zingone $\mathrm{F}$ et al. Post-thyroidectomy complications. The role of the device: bipolar vs ultrasonic device: Collection of data from 1,846 consecutive patients undergoing thyroidectomy. Am J Surg. 2016;212:116-21. Crossref

3. Muratli A, Erdogan N, Sevim S, Unal I, Akyuz S. Diagnostic efficacy and importance of fine-needle aspiration cytology of thyroid nodules. J Cytol. 2014;31:73-8. Crossref

4. Straccia P, Rossi ED, Bizzarro T, et al. A meta-analytic review of the Bethesda System for Reporting Thyroid Cytopathology: Has the rate of malignancy in indeterminate lesions been underestimated? Cancer Cytopathol. 2015;123:713-22. Crossref

5. Crippa S, Mazzucchelli L, Cibas ES, Ali SZ. The Bethesda System for reporting thyroid fine-needle aspiration specimens. Am J Clin Pathol 2010;134:343-4. Crossref

6. Svant R. Orell GFS. Orell \& Sterrett's Fine Needle Aspiration Cytology. 5th ed. Vielh SROaP, editor. New Delhi, India: Reed Elsevier India Private Limited.; 2012.482pp

7. Himakhun W, Chansom R, Aroonroch R, Pongtippan A. Fine needle aspiration of thyroid: a cyto-histopathological correlation in Ramathibodi Hospital. J Med Assoc Thai. 2012;95:S74-8. Crossref

8. Ko HM, Jhu IK, Yang SH et al. Clinicopathologic analysis of fine 
needle aspiration cytology of the thyroid. A review of 1,613 cases and correlation with histopathologic diagnoses. Acta Cytol 2003;47:72732. Crossref

9. Piromalli D, Martelli G, Del Prato I, Collini P, Pilotti S. The role of fine needle aspiration in the diagnosis of thyroid nodules: analysis of 795 consecutive cases. J Surg Oncol 1992;50:247-50. Crossref

10. Cap J, Ryska A, Rehorkova P, Hovorkova E, Kerekes Z, Pohnetalova D. Sensitivity and specificity of the fine needle aspiration biopsy of the thyroid: clinical point of view. Clin Endocrinol 1999;51:509-15. Crossref
11. Naz S, Hashmi AA, Khurshid A et al. Diagnostic accuracy of Bethesda system for reporting thyroid cytopathology: an institutional perspective. Int Arch Med 2014;7:46.Crossref 